\title{
Image-based CFD modelling of cerebral blood flow
}

\author{
Sekhane DJALAL ${ }^{* 1}$, Karim MANSOUR ${ }^{1,2}$ \\ ${ }^{1}$ LEMEAMED. Université des Frères Mentouri Constantine 1, Route d'Ain El bey, Constantine \\ Algeria \\ ${ }^{2}$ Faculté de Médecine de Constantine. Université Constantine 3 -Chalet des pins - Constantine \\ - Algeria
}

Corresponding author: E-mail: djalalsekhane@gmail.com

Keywords: MRI, CFD, Wall Shear Stress, Pressure

\begin{abstract}
Detailed knowledge of the hemodynamics in the cerebral blood tree is valuable not only for studying the mechanisms of alimentation in the cortex, but also to investigate the role of hemodynamics in the development of cerebrovascular pathologies. The aim of this work is to present preliminary data processing pipeline focussing on the extraction of a $3 \mathrm{D}$ patient specific smooth geometric model from magnetic resonance imaging (MRI) and estimate hemodynamic factors using computational fluid dynamics (CFD).
\end{abstract}

\section{Introduction}

Understand the blood flow in cerebral arterial tree have a major importance to understand the mechanisms of the transport of blood in the cortex. However, using Computational Fluid Dynamics (CFD) combined with medical imaging methods has increased with the big development known in these domains [1]. The use of this method seem to be necessary due to the limitation of the current imaging modalities to provide hemodynamic information and its capability to provide effective and safe information of blood flow inside the vessels[9,10]. In this work, a numerical simulation based on 3D Navies-stokes laws used to investigate the different hemodynamic parameters. The study concentrates on the Circle of Willis $(\mathrm{CoW})$, the base of cerebral vasculature network.

\section{Material and methods}

A. Vascular model :

Patient specific images of the circle of Willis were obtained from Magnetic Resonance Angiography (MRA), using 3D Time Of Flight (TOF) sequence with the following parameters: $\mathrm{TE}=3.1 \mathrm{~ms}, \mathrm{TR}=25 \mathrm{~ms}$ and slice thickness $=1.4 \mathrm{~mm}$.

Using 3D slicer software, a three dimensional geometry of the COW was created (fig1).

\section{B. Mathematical and blood model:}

The blood dynamics was modelled with the Navies-Stokes equation. In the case of a laminar flow, Navies-Stokes equation can be written as:

$$
\rho\left(\frac{\partial u}{\partial t}+u \cdot \nabla u\right)=-\nabla p+\nabla\left(\mu\left(\nabla u+(\nabla u)^{T}\right)-\frac{3}{2} \mu(\nabla \cdot u) I\right)+F
$$

Where $\rho$ is the density, $u$ the velocity and $\mu$ the dynamic viscosity.The blood is considered newtonian and incompressible with a density $\rho=1060 \mathrm{~kg} / \mathrm{m}^{3}$ and dynamic viscosity $\mu=4 \mathrm{mPa} . \mathrm{s}$ [2].

C. Numerical method:

To solve Navies-Stokes equation, we used COMSOL multiphysics, which uses finite elements method for spatial discretisation. The simulation was performed for three cycles and the typical flow rate is $4.6 \mathrm{ml} / \mathrm{s}$ [3].

The vessels walls was assumed rigidand no slip boundary condition was applied. 


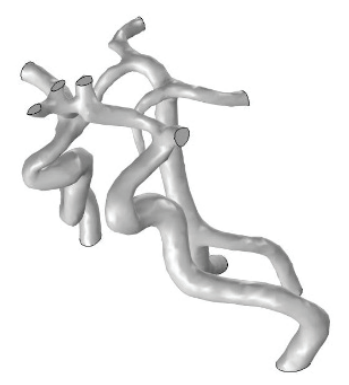

Fig1: Blood Vessels of the

\section{Results}

Fig 2 shows the pressure distribution at the systole time on a complete circle of Willis of a young health person, the pressure take values from 103 to $106 \mathrm{mmHg}$ distributed from high pressure in the internal carotids and vertebral arteries to low localised in anterior, middle and posterior cerebral arteries.

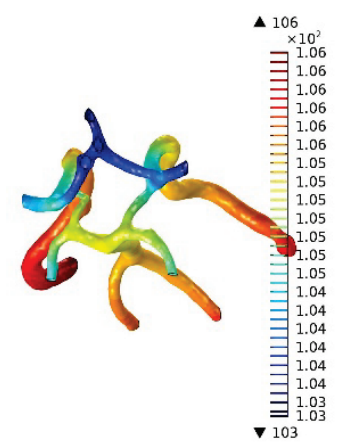

Fig 2: Pressure distribution inside the Circle of Willis

Fig 3 shows the wall shear stress (WSS) values resulting from the blood flow inside the different compartments of the circle of Willis, and obtained from the product of dynamic viscosity and the shear rate solution.

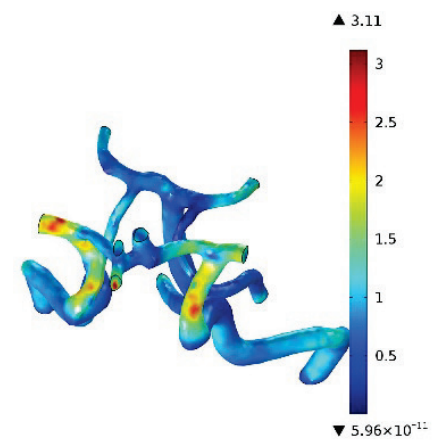

Fig 3: Wall shear stress map at the diastolic time.

Fig 4 shows the streamlines in the circle of Willis, it gives a cartography of the flow speed inside the different segments. 


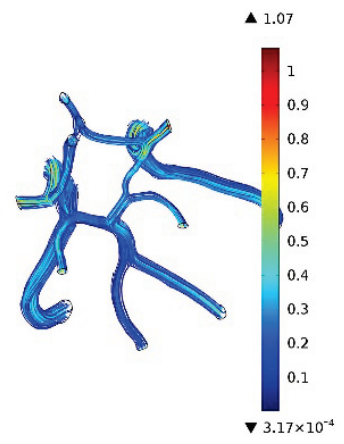

Fig 4: Stream lines inside the different segment of the circle of Willis

\section{Discussion}

In this study, we present results from the simulation of blood flow inside a complete circle of Willis, using CFD combined with patient specific images from a young person. Several hemodynamic parameters were used to investigate the effect of the blood flow on the major vessels of the brain. It is known that the WSS is involved in many pathophysiological processes related to vascular diseases [4], like aneurysms. It's suggested that high values of WSS may cause the initiation of an aneurysm, while low values facilitate the growth in the creation [5]. In our case, the values of WSS were low and the possibility of creation of an aneurysm is also low. However the highest values of WSS were localised near to bifurcation and at this location, creation of an aneurysm is eventual but still far. The pressure is also involved in many vascular diseases, like atherosclerosis defined as a deposition of plates on the vessel wall [6] and more aneurysms [4]. Where high values of pressure leads to cholesterol deposition on the carotid arteries [7]. Our results shows normal values of pressure [103$106 \mathrm{mmHg}]$, which keeps low probability for a vascular disease caused by the pressure.

\section{Conclussion}

In this study CFD based on patient specific images was performed on a healthy young person to investigate the hemodynamic factors. The hemodynamic factors investigated have shown no abnormal values on the different compartments, no risk for cerebro-vascular disease has been found.

\section{References}

[1] Juan Cebral, Image-Based CFD Modelling of Cerebral Aneurysms, Computational Biomechanics for Medicine: Deformation and Flow VIII, 140p. , 2012.

[2] Yufeng Hua et al.Influence of Parent Artery Segmentation and Boundary Conditions on HemodynamicCharacteristics of Intracranial Aneurysms. Yonsei Medical journal. 2015. http://dx.doi.org/10.3349/ymj.2015.56.5.1328

[3] J. Xiang, A. Siddiqui, and H. Meng, "The effect of inlet waveforms on computationalhemodynamics of patient-specific intracranial aneurysms," Journal of biomechanics, vol. 47, no. 16, pp. 3882-3890, 2014. http://dx.doi.org/10.1016/j.jbiomech.2014.09.034

[4] M. Cibis, W. V. Potters, F. J. Gijsen et al., "Wall shear stress calculations based on 3D cine phase contrast MRI and computational fluid dynamics: a comparison study in healthy carotid arteries," NMR in Biomedicine, vol. 27, no. 7, pp. 826-834, 2014. http://dx.doi.org/10.1002/nbm.3126

[5] M. Shojima, M. Oshima, K. Takagi et al., "Magnitude and role of wall shear stress on cerebral aneurysm computational fluid dynamic study of 20 middle cerebral artery aneurysms," Stroke, vol. 35, no. 11, pp. 2500-2505,2004. http://dx.doi.org/10.1161/01.STR.0000144648.89172.0f 
[6] N. Westerhof, N. Stergiopulos, and M. I. Noble, Snapshots of hemodynamics: an aid for clinical research and graduate education: Springer Science \& Business Media, 2010.

http://dx.doi.org/10.1007/978-1-4419-6363-5

[7] P. Sobieszczyk, and J. Beckman, "Carotid artery disease," Circulation, vol. 114, no. 7, pp. e244e247, 2006. http://dx.doi.org/10.1161/CIRCULATIONAHA.105.542860

[8] T. David, S. Moore. "Modeling perfusion in the cerebral vasculature". Medical Enginnering\& Physics 30, (2008) 1227-45. http://dx.doi.org/10.1016/j.medengphy.2008.09.008

[9] V. C. Rispoli, J. F. Nielsen, K. S. Nayak et al., "Computational fluid dynamics simulations of blood flow regularized by 3D phase contrast MRI," Biomedical engineering online, vol. 14, no. 1, pp. 110, 2015. http://dx.doi.org/10.1186/s12938-015-0104-7

[10] Y. Hua, J. H. Oh, and Y. B. Kim, "Influence of Parent Artery Segmentation and Boundary Conditions on Hemodynamic Characteristics of Intracranial Aneurysms," Yonsei medical journal, vol. 56, no. 5, pp. 1328-1337, 2015. http://dx.doi.org/10.3349/ymj.2015.56.5.1328 